\title{
Líquido dializante con citrato frente a acetato en pacientes en hemodiafiltración on-line de alta eficacia: parámetros a estudio
}

\author{
Sonia Aznar Barbero, Ramón Bel Cegarra, Josefa Chica Arellano, Aránzazu Martínez Martínez, Mª Magdalena \\ Badallo Mira, Mercedes Sánchez Clavel, Susana Carbonell Olivares, Santiago Martínez Domingo \\ Enfermero/as de la Unidad de Hemodiálisis del Hospital Santa Lucía. Cartagena. Murcia
}

\section{Resumen}

Introducción: El líquido dializante con acetato se reporta menos biocompatible, generando estrés oxidativo e inflamación. Entre los beneficios asociados al citrato destacan sus propiedades antiinflamatorias y antioxidantes, poder anticoagulante, quelante del calcio iónico, efecto tampón y mejora la tolerancia a la sesión.

Objetivo: Comparar parámetros nutricionales e inflamatorios, eficacia dialítica, estatus ácido-base, tolerancia y balance de calcio en hemodiafiltración en línea empleando líquido dializante con citrato frente a líquido dializante con acetato.

Material y métodos: Estudio prospectivo cruzado sobre población en hemodiafiltración en línea de 24 semanas. Inician 35 pacientes (finalizan 33), con 57, 4 años, 55\% hombres, 54,1 meses de permanencia. $79 \%$ portadores de fistula arteriovenosa. Se analizan 2376 sesiones. EI estudio tiene dos fases. Variables: albúmina, PCR, Beta 2 microglobulinas (B2MCG), ángulo de fase (AF), masa celular corporal $(B C M)$, Kt, volumen de reinfusión, PTTA, tiempo de hemostasia, estado de cámaras y dializador, $\mathrm{pH}$, bicarbonato, calcio iónico, sesiones con hipotensión y calambres. Análisis estadístico SPSS 13.0. Contraste de hipótesis mediante T-Student y W de Wilcoxon para variables cuantitativas. Chi-cuadrado cualitativas. Significación estadística $p<0,05$.

Resultados: Diferencias significativas $(p<0,001)$ en albúmina, PCR, y B2MCG. BCM $(p=0,001), A F(p=0,002)$ y Kt $(p<0,001)$ mayores con líquido de diálisis con citrato. Bicarbonato postdiálisis menor $(p<0,001)$ con líquido de diálisis con citrato. Calcio iónico pre y postdiálisis $(p=0,007$ y $p<0,001$ respectivamente) menores con líquido de diálisis con citrato.

Sonia Aznar Barbero

C/ Agua del Molino $n^{\circ} 35$

30310. Los Dolores. Cartagena. Murcia

E-mail: soniazbar@hotmail.com
Conclusiones: La diálisis con citrato se muestra en nuestra serie más biocompatible y con menor inflamación, mejora los parámetros nutricionales analíticos y por impedancia e incrementa la eficacia dialítica. Reduce el bicarbonato postdiálisis. Tanto pre como postdiálisis el calcio iónico es menor, permaneciendo estable.

PALABRAS CLAVE

- HEMODIAFILTRACIÓN ON-LINE

- LÍQUIDO DIALIZANTE

- CITRATO

- BIOCOMPATIBLE

- EFICACIA DIALÍTICA

Citrate- versus acetate- based dialysate in patients in high-efficiency online hemodiafiltration: parameters to be studied

\begin{abstract}
Introduction: Liquid dialysate with acetate (LDA) reported less biocompatible, generating oxidative stress and inflammation. Perceived benefits include its anti-inflammatory citrate buffer effect and antioxidant properties, anticoagulant power, chelating ionized calcium, and improves tolerance to the session.
\end{abstract}

Objective: To compare nutritional and inflammatory parameters, dialysis efficiency, acid-base status, tolerance and calcium balance in on-line hemodiafiltration (OLHDF) using citrate dialysate (LDC) versus LDA.

Methods: Prospective cross OLHDF population in 24 weeks. Begins 35 patients (33 finish), with 57.4 years, $55 \%$ male, 54.1 months of permanence. $79 \%$ carriers arteriovenous fistula. 2376 sessions are analyzed. The study has two phases. Variables: albumin, CRP, Beta 2 microglobulin (B2MCG), phase angle (PA), body cell mass $(B C M)$, Kt, reinfusion volume, PTTA, hemostasis 
time, state cameras and dialyzer, $\mathrm{pH}$, bicarbonate, calcium ion, sessions with hypotension and cramps. Statistical analysis SPSS 13.0. Hypothesis testing using T-Student and Wilcoxon $\mathrm{W}$ for quantitative variables. Chi-squared qualitative. Statistical significance $p$ $<0.05$.

Results: Significant differences $(p<0.001)$ in albumin, CRP, and B2MCG. BCM $(p=0.001), A F(p=0.002)$ and $\mathrm{Kt}(p<0.001)$ higher with LDC. Lower post-dialysis bicarbonate $(p<0.001)$ with LDC. Calcium ion pre and post-dialysis ( $p=0.007$ and $p<0.001$ respectively) with lower LDC.

Conclusion: Dialysis citrate shown in our series more biocompatible and less inflammation, improves nutritional parameters and analytical impedance and increases efficiency dialysis. Reduces post-dialysis bicarbonate. Both pre-and post-dialysis ionized calcium is lower, remaining stable.

\section{KEYWORDS}

- ON-LINE HEMODIAFILTRATION

- DiALYSATE FLUID

- CITRATE

- BIOCOMPATIBLE

- DIALYSIS EFFICIENCY

\section{Introducción}

El líquido dializante con acetato (LDA) se ha mostrado como menos biocompatible, generando estrés oxidativo y la consiguiente inflamación por la liberación de citoquinas proinflamatorias ${ }^{1}$. Estudios recientes indican que la presencia de acetato en el líquido de diálisis puede ser un factor importante en la intolerancia de la diálisis siendo parte responsable de síntomas intradialíticos como fatiga, hipotensión, mareos, etc²

Por el contrario, la diálisis con líquido dializante con citrato (LDC) se ha descrito con propiedades antiinflamatorias y antioxidantes ${ }^{3}$, asociándose su uso con una disminución de los niveles plasmáticos de proteína Creactiva $(P C R)^{4,5}$.

El empleo de líquido dializante con citrato puede incrementar la eficacia del dializador dado su conocido poder anticoagulante ${ }^{6}$, ya que actúa de forma temprana sobre el calcio en la cascada de la coagulación, así como disminuir la precipitación de sales cálcicas al actuar como quelante del calcio iónico. Además de estos beneficios, consigue una mejoría del equilibrio ácido-base dado su efecto tampón y una mayor tolerancia a la sesión de diá- lisis consecuencia de una mayor estabilidad cardiovascular ${ }^{7}$.

El objetivo de este estudio es comparar el comportamiento de diversos parámetros con ambos líquidos dializantes en pacientes en hemodiafiltración on-line (HDFOL) de alta eficacia: parámetros nutricionales e inflamatorios, eficacia dialítica, estatus ácido-base, la tolerancia y el balance de calcio.

\section{Material y métodos}

Estudio prospectivo cruzado sobre población prevalente en hemodiafiltración On-Line posdilucional con más de 3 meses de permanencia en la técnica, de 24 semanas de duración. Inician 35 pacientes de los que finalizan 33 (1 trasplante, 1 exitus), con 57,4 años de media, 55\% hombres, 54,1 meses de permanencia de media. La etiología glomerular es la más frecuente $(28 \%)$, seguida de la vascular (18\%). El $79 \%$ de los pacientes son portadores de fístula arteriovenosa, $18 \%$ de catéter tunelizado y $3 \%$ de prótesis.

Criterios de inclusión: pacientes mayores de 18 años en tratamiento con hemodiafiltración on-line posdilucional portadores de un acceso vascular estable que alcance un flujo sanguíneo no inferior a $350 \mathrm{ml} / \mathrm{min}$. Todos los pacientes dan consentimiento expreso.

Criterios de exclusión: Negativa a participar en el estudio, intolerancia o alergia al citrato e hipocalcemia sintomática.

Se analizan un total de 2.376 sesiones. El estudio se realiza en dos fases:

- FASE 1: Durante las primeras 12 semanas 17 pacientes se dializan con líquido de diálisis con citrato (LDC) (1 mmol/l), permaneciendo los otros 16 con acetato (LDA) (3 mmol/l.).

- FASE 2: Las 12 semanas siguientes los pacientes se cruzan.

Los pacientes con LDA se dializan con dos formulaciones de calcio: 1,25 ( $42,4 \%$ de los pacientes) y 1,50 $(57,6 \%)$. LDC con formulaciones 1,50 y $1,65 \mathrm{mmol} / \mathrm{l}$. No se realiza ninguna otra variación en las sesiones (ni en dializador ni en dosis de heparina).

Los dializadores usados son membranas de poliamida de $2,1 \mathrm{~m}^{2}$.

El flujo sanguíneo (Qb) se mantiene estable durante todo el estudio individualizándose en cada paciente, manteniéndose siempre superior a $350 \mathrm{ml} / \mathrm{min}$. El Flujo de 
líquido dializante (Qd) se establece en $500 \mathrm{ml} / \mathrm{min}$. El tiempo de las sesiones se fija en 240 minutos efectivos.

Las variables a estudio son: albúmina, PCR, Beta 2 microglobulinas (B2MCG), parámetros de impedancia (monofrecuencia): ángulo de fase (AF) y masa celular corporal (BCM), Kt, volumen de reinfusión (VTR), PTTA, tiempo de hemostasia, estado de cámaras y dializador, pH, bicarbonato y calcio iónico ( $\mathrm{Ca}++$ ) pre y postdiálisis, sesiones con hipotensión, sesiones con calambres.

Las determinaciones analíticas se realizan del siguiente modo:

-Albumina, PCR y B2 microglobulinas: semanas 0, 12 y 24.

- Calcio iónico predialisis: semanas $0,2,4,6,8,10$, $12,14,16,18,20,22,24$.

- Bicarbonato, pH, PC02, EB, Calcio iónico y Coagulación (PTTA y PT) postdiálisis: 0, 2, 4, 6, 8, 10, $12,14,16,18,20,22,24$.

La impedancia monofrecuencia se realiza prediálisis las semanas 0,12 y 24 .

El tiempo de hemostasia se mide mediante la siguiente escala: 0-5 min, 5-10 min, 10-15 min y más de 15 minutos.

Para medir el estado de las cámaras y el dializador usamos la escala: 0-Coagulado, 1-Sucio, 2-Intermedio, 3-Limpio.

El análisis estadístico se realiza mediante el programa SPSS 13.0 para Windows. Para el contraste de hipótesis empleamos el Test de Kolmogoroff-Smirnoff para la comprobación de normalidad. Utilizamos T-Student (para muestras relacionadas) y $\mathrm{W}$ de Wilcoxon para variables cuantitativas y Chi-cuadrado de Pearson para cualitativas.

Como variables cuantitativas empleamos la media, desviación estándar y rango y como variables cualitativas las frecuencias y porcentajes.

Consideramos significación estadística $p<0,05$.

\section{Resultados}

Se aprecian diferencias significativas $(p<0,001)$ en albúmina ( $\operatorname{lDC} 3,87 \pm 0,31$ vs LDA 3,64 $\pm 0,34$ ), PCR (LDC 0,8 $\pm 0,75$ vs LDA $1,92 \pm 2,56 \mathrm{ng} / \mathrm{ml}$ ), y B2MCG ( $L D C 22,51 \pm 6,20$ vs LDA $(25,02 \pm 6,05 \mathrm{mg} / \mathrm{dl})$.

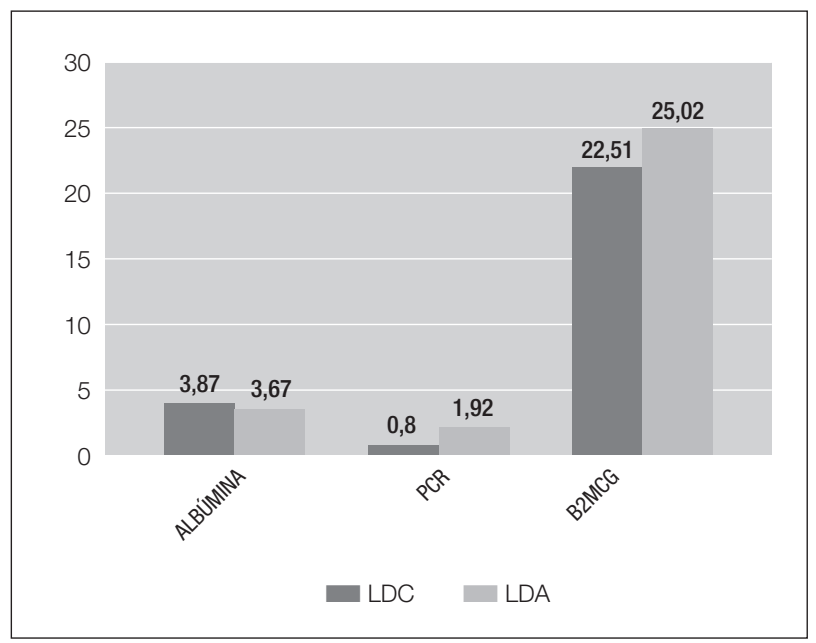

Figura 1. Albúmina, PCR y 32 Microglobulinas.

En relación con los parámetros de bioimpedancia tanto BCM $(p=0,001)$ como AF $(p=0,002)$ son significativamente mayores con LDC $(27,65 \pm 8,08$ y 5,69 $\pm 1,31$, respectivamente) que con $\operatorname{LDA}(25,01 \pm 7,15$ y 5,06 $\pm 0,91$ respectivamente).

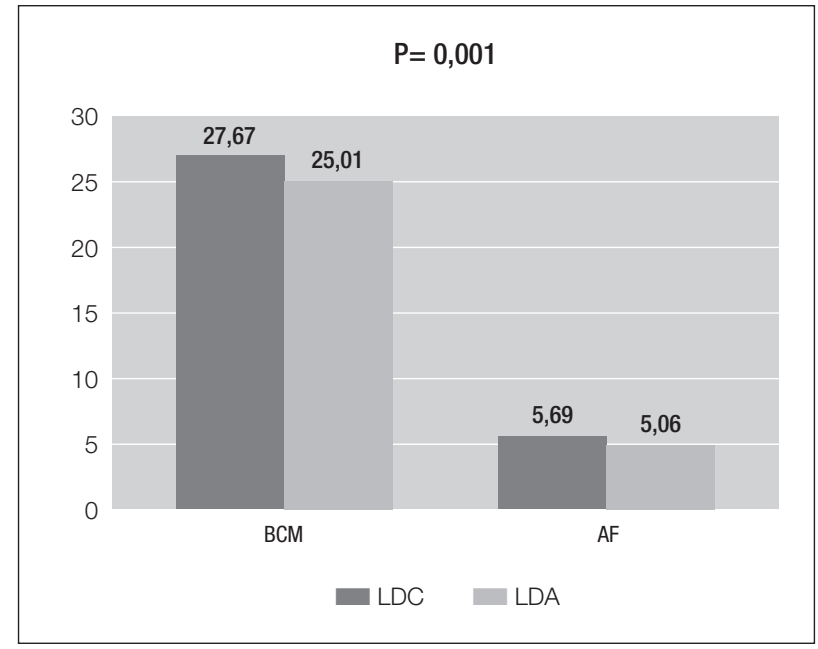

Figura 2. Masa Celular Corporal y Ángulo de Fase.

El Kt es significativamente $(p<0,001)$ mayor con LDC que con $\operatorname{LDA}(58,44 \pm 3,37$ versus $56,94 \pm 3$,18 litros), sin diferencias en flujo sanguíneo $(386,01 \pm 23,41$ versus $382,92 \pm 32,97 \mathrm{ml} / \mathrm{min})$ ni tiempo efectivo $(252,64$ $\pm 3,27$ versus $252,89 \pm 3,21$ minutos). No se encuentran diferencias en VTR. 


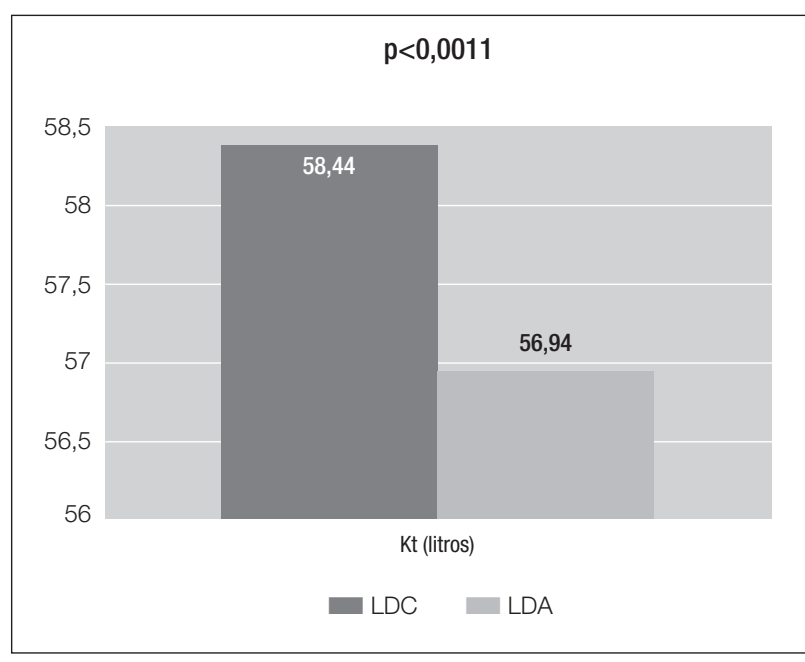

Figura 3. Kt.

EI PTTA $(1,53 \pm 0,37$ versus $1,49 \pm 0,36)$ y tiempo de hemostasia ( $14,37 \pm 5,01$ versus $14,19 \pm 5,35$ minutos) se muestran mayores con LDC, pero sin que estas diferencias sean significativas. Las cámaras y el dializador quedan más limpios usando LDC en comparación con LDA pero sin diferencias significativas.

No se aprecian diferencias significativas en $\mathrm{pH}$ pre, $\mathrm{pH}$ pos y bicarbonato predialisis. El bicarbonato posdialisis es significativamente $(p<0,001)$ menor con $\operatorname{LDC}(27,97$ $\pm 0,70 \mathrm{mmol} / \mathrm{l})$ que con LDA $(29,32 \pm 0,87 \mathrm{mmol} / \mathrm{l})$. Tanto el calcio iónico pre $(4,52 \pm 0,28$ versus $4,61 \pm 0,31$ $\mathrm{mg} / \mathrm{dl})$ como el calcio iónico pos $(4,51 \pm 0,21$ versus $4,93 \pm 0,25 \mathrm{mg} / \mathrm{dl})$ son significativamente $(p=0,007$ y $p<$ 0,001 , respectivamente) menores con LDC.

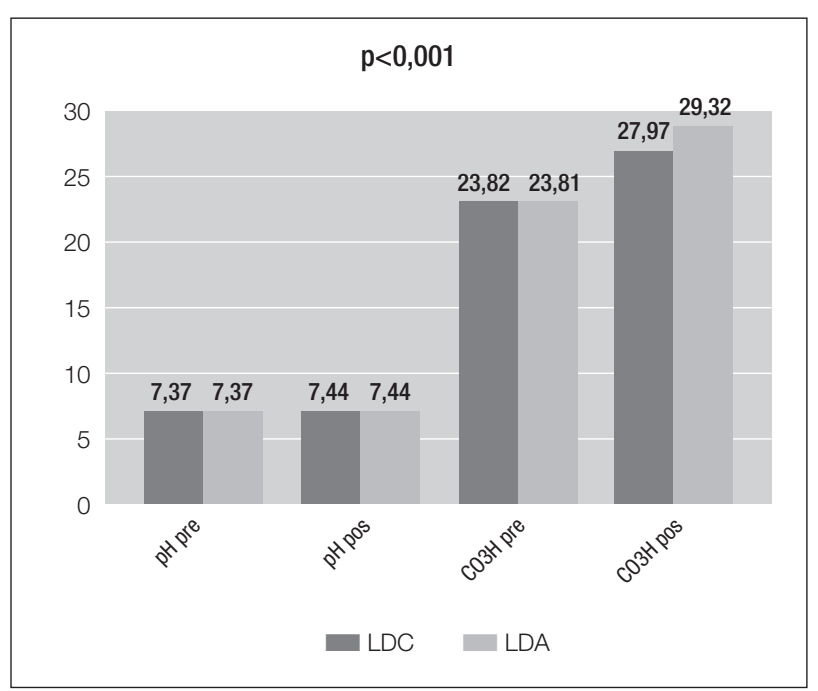

Figura 4. pH y Bicarbonato.

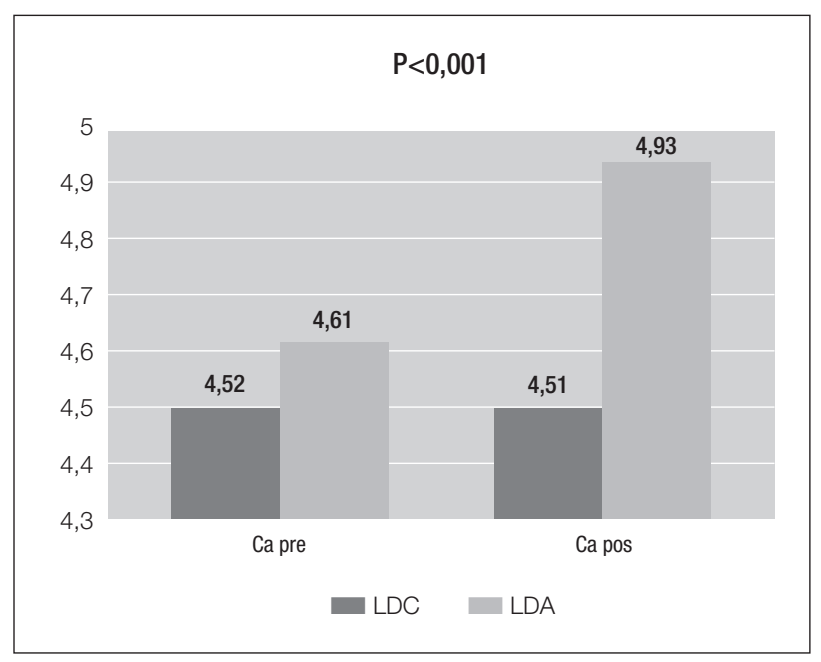

Figura 5. Calcio pre y post.

Aunque no existen diferencias significativas, el número de sesiones con hipotensión $(3,94 \pm 6,58$ con LDC versus $2,85 \pm 2,76$ con LDA) son mayores con LDC y el número de sesiones con calambres son mayores con LDA ( 1,81 $\pm 3,6$ con LDC versus $2,06 \pm 2,7$ con LDA).

\section{Discusión}

La diálisis con LDC resulta eficaz para modular la respuesta inflamatoria de nuestros pacientes dando lugar a niveles reducidos de PCR coincidiendo con los resultados de Matsuyama et $\mathrm{al}^{4}$, y menores niveles de lipoproteínas de baja densidad (B2MCG) limitando el estrés oxidativo asociado a la diálisis ${ }^{8}$.

En relación con el aumento de eficacia dialítica al usar citrato en el baño de diálisis nuestros datos muestran un aumento significativo en la dosis de diálisis encontrando resultados similares en los estudios de Kossmann et $\mathrm{al}^{8}$, Ahmad et $\mathrm{al}^{9}$ y Gabutti et al ${ }^{7}$ (éste último sin significación estadística). Sin embargo, otros autores no encuentran diferencias en éste parámetro ${ }^{10}$. El citrato en el líquido de diálisis produce un aumento en la eficacia dialítica debido fundamentalmente a dos factores, por un lado, como consecuencia de la reducción de la trombogenicidad y por otro, por la disminución de la precipitación de sales cálcicas, obteniendo en suma una mejor permeabilidad de las fibras del dializador.

El citrato es un metabolito con conocido poder anticoagulante capaz de reducir el potencial trombogénico del circuito extracorpóreo al limitar la disponibilidad de iones de calcio, que son un factor clave en la cascada de la coagulación, mejorando de éste modo el rendimiento 
de la máquina de diálisis. Nuestros resultados sugieren una reducción de la trombogenicidad al usar un LDC, encontrando un aumento del PTTA sin que éste sea significativo como ocurre en otros estudios (Calabresse et al. Personal Communication 50th ERA-EDTA Congress, Turkey 2013). En nuestra serie el circuito extracorpóreo queda más limpio al usar LDC, lo cual podría explicarse por un efecto local de disminución de la coagulación a nivel del dializador ${ }^{8}$.

En nuestra muestra el bicarbonato postdiálisis resulta significativamente menor con LDC coincidiendo con el estudio de Calabresse et al (Personal Communication 50th ERA-EDTA Congress, Turkey 2013), aunque se mantiene sin diferencias predialisis en contraposición con los resultados de Gabutti et al ${ }^{7}$. También los resultados de niveles más bajos de calcio iónico coinciden con los mostrados en otros estudios ${ }^{7}$ pese a la corrección del calcio en el baño de diálisis al emplear un líquido dializante con citrato. Dado el poder quelante del citrato hemos empleado formulaciones de calcio mayores en el periodo de LDC (pasando de 1,25 LDA a 1,50 LDC y de 1,50 LDA a 1,65 LDC) asimilando los resultados de otros autores como Nilsson et al (Personal Communication 49th ERA-EDTA Congress, France 2012) y Steckiph et al (Personal Communication 50th ERA-EDTA Congress, Turkey 2013).

El uso de un líquido de diálisis con citrato conlleva una reducción del malestar en los pacientes debido a la ausencia de acetato ${ }^{11}$, que se ha relacionado con diversos efectos clínicos adversos secundarios a su uso (dislipemia, depresión miocárdica, producción de citoquinas, vasodilatación e hipoxia), además de estar asociado a síntomas intradialíticos como mareo, nauseas, fatiga, hipotensión, etc. En nuestra serie no encontramos diferencias significativas en cuanto a hipotensiones y calambres entre los periodos de LDC y LDA, aunque el hecho de encontrar un mayor número de hipotensiones en el periodo de citrato puede estar relacionado con la inseguridad que vive el paciente ante el uso de materiales nuevos desconocidos por él hasta el momento.

\section{Conclusiones}

La diálisis con citrato se muestra en nuestra serie más biocompatible (menor B2MCG) y con menor inflamación medida por PCR. Además, mejora los parámetros nutricionales analíticos (albúmina) y por impedancia (BCM y AF). Estos datos se asemejan a los obtenidos por otros autores que describen una reducción significativa de los niveles de proteína C-reactiva, con efectos positivos sobre parámetros nutricionales concomitantes.
El uso de LDC en HDFOL mejora un $2,6 \%$ la eficacia dialítica. Además, reduce el bicarbonato postdiálisis, haciéndolo más fisiológico. Pese a la corrección del calcio en el baño, tanto pre como postdiálisis el calcio iónico es menor, permaneciendo estable, con LDC. El empleo de LDC no mejora la tolerancia en HDFOL.

Estos resultados deben ser confirmados con estudios con un mayor tamaño muestral y un mayor periodo de seguimiento.

Recibido: 12 Agosto 2014

Revisado: 18 Agosto 2014

Modificado: 20 Agosto 2014

Aceptado: 20 Agosto 2014

\section{Bibliografía}

1. Pizzarelli F, Cerrai T, Dattolo P, Ferro G. On-line haemodiafiltration with and without acetate. Neprhol Dial Transplant. 2006;21(6):1648-51.

2. Lindsay RM, Heidenheim PA, Nesrallah G, Garg AX, Suri R. Minutes to recovery after a hemodialysis session: a simple health-related quality of life question that is reliable, valid, and sensitive to change. Clin J Am Soc Nephrol. 2006;1(5):952-959.

3. Bryland A, Wieslander A, Carlsson 0, Hellmark $T$ and Godaly G. Citrate treatment reduces endothelial deah and inflammation under hyperglycaemic conditions. Diab Vasc Dis Res. 2012;9(1):42-51.

4. Matsuyama K, Tomo T, Kadota J. Acetate-free blood purification can impact improved nutritional status in hemodialysis patients. J Artif Organs. 2011;14(2):112-9.

5. Higuchi T, Yamamoto C, Kuno T, Okada K, Soma M, Fukuda N, et al. A comparison of bicarbonate hemodialysis, hemodiafiltration, an acetate-free biofiltration on cytokine production. Ther Apher Dial. 2004;8(6):460-7.

6. Sands JJ, Kotanko $P$, Segal JH, Ho CH, Usvat $L$, Young $A$, et al. Effects of citrate acid concentrate on heparin $\mathrm{N}$ requirements and hemodialysis adequacy: a multicenter, prospective noninferiority trial. Blood Purif. 2012;33(1-3):199-204. 
7. Gabutti L, Lucchini B, Marone $C$, Alberio $L$ and Burnier M. Citrate- vs. acetate-based dialysate in bicarbonate haemodialysis: consequences on haemodynamics, coagulation, acid-base status, and electrolytes. BMC Nephrol. 2009;10:7.

8. Kossmann RJ, Gonzales A, Callan R, Ahmad S. Increased efficiency of hemodialysis with citrate dialysate: a prospective controlled study. Clin J Am Soc Nephrol. 2009;4(9):1459-64.

9. Ahmad S, Callan R, Cole JJ, Blagg CR. Dialysate made from dry chemicals using citric acid increases dialysis dose. Am J Kidney Dis. 2000; 35 (3): 493-9.
10. Kuragano $T$, Kida $A$, Furuta $M$, Yahiro $M$, Kitamura $R$, Otaki $Y$, et al. Effects of acetate-free citratecontaining dialysate on metabolic acidosis, anemia, and malnutrition in hemodialysis patients. Artif Organs. 2012 Mar;36(3):282-90.

11. Daimon S, Dan K, Kawano M. Comparison of acetate-free citrate hemodialysis and bicarbonate hemodialysis regarding the effect of intra-dialysis hypotension and postdialysis malaise. Ther Apher Dial. 2011;15(5):460-5. 